\title{
A Search for Solar Wind Velocity Changes Between 0.7 and 1 AU
}

\author{
D. S. INTRILIGATOR ${ }^{1}$ \\ Physics Department, University of Southem California. Los Angeles, California 90007 \\ M. NeUgebauer
}

Jet Propulsion Laboratory, California Institute of Technology, Pasadena, California 91103

\begin{abstract}
Simultaneous observations of the solar wind velocity as measured at the Pioneer 9 and Ogo 5 spacecraft during five solar rotations in 1968 and 1969 are presented. During this time, Pioneer 9 was traveling in toward the sun to approximately $0.7 \mathrm{AU}$ while the earth orbiter Ogo 5 was spending long periods in the interplanetary medium. A comparison of the 3-hour averages of solar wind velocity obtained at both spacecraft indicates that the same basic solar wind velocity structure was seen at both spacecraft. There was no statistically significant dependence of average velocity on radial distance from the sun. The amplitude of variations about the average velocity apparently decreased with increasing distance from the sun; some but probably not all of this decreased variation is consistent with the exchange of momentum between high-velocity and low-velocity streams. The correlation coefficient of pairs of velocity averages computed from a corotation model decreases with increasing distance between the two spacecraft.
\end{abstract}

This paper presents observations concerning radial variations of the solar wind velocity between 0.7 and $1 \mathrm{AU}$ in late 1968 and early 1969. During this time the Pioneer 9 spacecraft traveled in toward the sun to approximately $0.7 \mathrm{AU}$ while the earth-orbiting satellite Ogo 5 spent long periods in the interplanetary medium. The Pioneer and Ogo data presented here are from the solar wind plasma spectrometers built by Ames Research Center (for Pioneer) and the Jet Propulsion Laboratory (for Ogo). The instruments have been described in reports by Intriligator et al. [1969], Graham and Vescelus [1967], and Neugebauer [1970, 1971]. The experimental uncertainties in each absolute value of velocity are approximately $\pm 5 \%$ for Pioneer and $\pm 3 \%$ for Ogo. About half of these uncertainties are due to uncertainties in the absolute velocity scales with the other half arising from the data-fitting techniques used. Thus relative shifts in velocity are more accurately determined than are the absolute values at the two spacecraft. Discrepancies larger than these uncertainties could arise in time averages of velocity if there are different times of occurrence and lengths of data gaps in the two sets of data. This effect can be important when Ogo spends an appreciable fraction of a 3-hour interval inside the earth's bow shock.

The Pioneer 9 trajectory relative to the earth and sun is shown in Figure 1 for the 4 months following launch on November 11, 1968. During this period, Ogo 5 moved between geocentric distances of $11,000 \mathrm{~km}$ (perigee) and $149,000 \mathrm{~km}$ (apogee) with a period of 62 hours. The solar ecliptic longitude of the Ogo apogee decreased from $+78^{\circ}$ to $-47^{\circ}$.

The Pioneer and Ogo velocity measurements are compared by using each 3-hour average of velocity at Pioneer to compute the time at which this plasma should arrive at Ogo according to a corotation model, for which

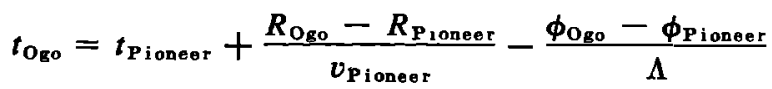

where $t$ is the time of observations, $R$ is distance from the sun, $v$ is solar wind velocity, $\phi$ is heliographic longitude, and $\Lambda$ is

1 This work was begun at the California Institute of Technology, Pasadena. California 91125 .

Copyright $@ 1975$ by the American Geophysical Union. the synodic rotation rate of the sun, which is assumed to equal $0.5515 \mathrm{deg} / \mathrm{h}$. The appropriate 3-hour average at Ogo is computed as the average of the hourly averages for the hour containing the predicted arrival time, the previous hour, and the following hour. A total of $\mathbf{4 0 3}$ pairs of 3-hour averages are thus formed; roughly half (230) of these pairs of averages were obtained when Pioneer was closer to the sun than $0.9 \mathrm{AU}$.

Table 1 summarizes the differences between the solar wind velocity at Ogo and Pioneer as determined from these corotated pairs of 3-hour averages. The data are divided according to whether Pioneer is closer to or further from the sun than 0.9 AU. Part 2 of Table 1 shows that for both $\boldsymbol{R}_{\text {Ploneer }}$ $>0.9 \mathrm{AU}$ and $R_{\text {Ploneer }}<0.9 \mathrm{AU}$ the average velocity at Ogo was equal to that at Pioneer within the absolute uncertainties of the measurements; this finding is in agreement with the approximately constant average velocity observations by Mariner 2 between 1.0 and $0.7 \mathrm{AU}$ [Neugebauer and Snyder, 1966]. Part 2 also shows that the standard deviations of the 3hour averages are greater at Pioneer than at Ogo; thus the variation of velocity about the mean evidently decreases with

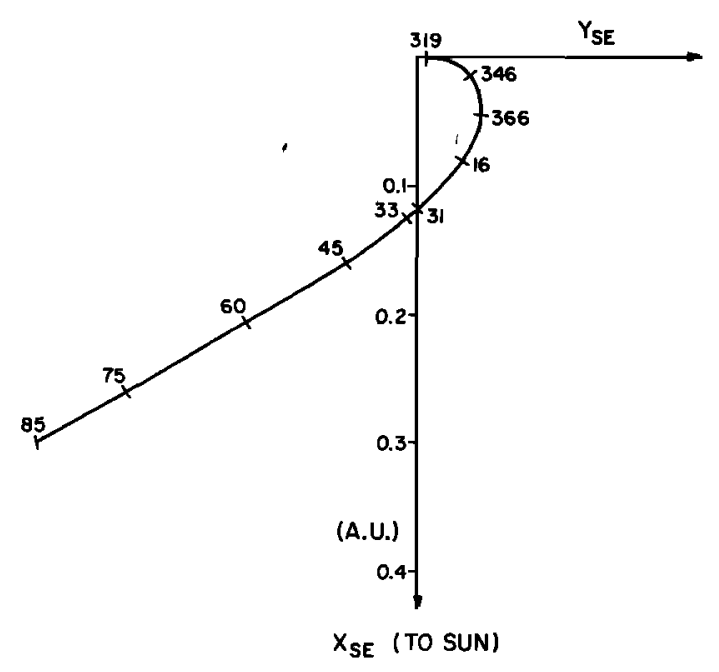

Fig. 1. Geocentric ecliptic projection of the Pioneer 9 trajectory from launch on November 11, 1968 (day 316), through March 16 (day 75), 1969. 
TABLE 1. Summary of Pairs of 3-Hour Averages

\begin{tabular}{|c|c|c|}
\hline & $R_{\text {Ploneer }} \geq 0.9 \mathrm{AU}$ & $R_{\text {Ploneer }} \leq 0.9 \mathrm{AU}$ \\
\hline $\begin{array}{l}\text { 1. Number of pairs of 3-hour averages } \\
\text { 2. Average velocity, } \mathrm{km} / \mathrm{s}\end{array}$ & 173 & 230 \\
\hline$v_{\text {PIoneer }}$ & $432 \pm 70$ & $459 \pm 84$ \\
\hline $\begin{array}{l}\text { vogo } \\
\text { 3idth of histogram: } 95 \% \text { value minus } 5 \% \text { value, } \\
\mathrm{km} / \mathrm{s}\end{array}$ & $432 \pm 66$ & $447 \pm 69$ \\
\hline $\begin{array}{l}\text { Pioneer } \\
\text { Ogo }\end{array}$ & $\begin{array}{l}560-324=236 \\
547-326=221\end{array}$ & $\begin{array}{l}618-361=257 \\
589-369=220\end{array}$ \\
\hline 4. Differences between Ogo and Pioneer & & \\
\hline $\begin{array}{l}\left\langle v_{\mathrm{P} \text { Ioneer }}-v_{\mathrm{Ogo}}\right), \mathrm{km} / \mathrm{s} \\
\left\langle\left|v_{\mathrm{Ploneer}}-v_{\mathrm{Ogo}}\right|\right\rangle, \mathrm{km} / \mathrm{s} \\
\left\langle t_{\text {Ogo }}-t_{\text {PIoneer }}\right\rangle, \text { hours } \\
\text { Range of } t_{\text {Ogo }}-t_{\text {Ploneer }}, \text { hours } \\
\text { Correlation coefficient between } v_{\mathrm{Ogo}} \text { and } v_{\mathrm{Ploneer}} \\
\text { Distance between spacecraft, AU }\end{array}$ & $\begin{array}{c}0.3 \\
15.7 \\
8.2 \\
0.7 \text { to } 14.6 \\
0.96 \\
0 \text { to } 0.09\end{array}$ & $\begin{array}{c}11.3 \\
31.6 \\
3.4 \\
-22.0 \text { to } 14.6 \\
0.84 \\
0.09 \text { to } 0.40\end{array}$ \\
\hline
\end{tabular}

increasing distance from the sun. This second conclusion is supported by the information in part 3 of Table 1 , which shows that the velocity histograms are narrower at $1 \mathrm{AU}(\mathrm{Ogo})$ than at a distance closer to the sun (Pioneer), with the difference increasing with the radial separation of the two spacecraft. These findings are in agreement with those of Collard and Wolfe [1974], who compared the solar wind velocity measured once per day at Pioneer $10(4.1-5.0 \mathrm{AU})$ with similar measurements at Pioneer 11 (1.0-2.7 AU). Collard and Wolfe suggest that the decrease in the amplitude of velocity variations with increasing distance from the sun is caused by the exchange of momentum between the high-speed and the low-speed streams in the solar wind.

Figure 2 shows the relation of the Ogo 5 and Pioneer 9 velocity differences to the solar wind stream structure. The bars in this figure are 3-hour averages of velocity observed at
Pioneer 9, and the circles are the corresponding values at Ogo computed when both Pioneer and Ogo data are available at the proper times (as computed from the corotation model).

The high-velocity stream observed on days $24-28$ is an example consistent with Collard and Wolfe's suggestion of momentum exchange and damping of the stream structure. Figure 2 also shows, however, that probably some of the apparent decrease of velocity variations with distance shown in Table 1 is due to chance and to the inaccuracy of the corotation model rather than to momentum exchange between highvelocity and low-velocity streams. For example, consider the data at the very end of day 59 in Figure 2 when Ogo did not see the $>700-\mathrm{km} / \mathrm{s}$ plasma observed by Pioneer 9 . The correct explanation of this large difference between the Ogo and Pioneer observations is probably the breakdown of the corotation model rather than the damping of the stream. If one assumes a

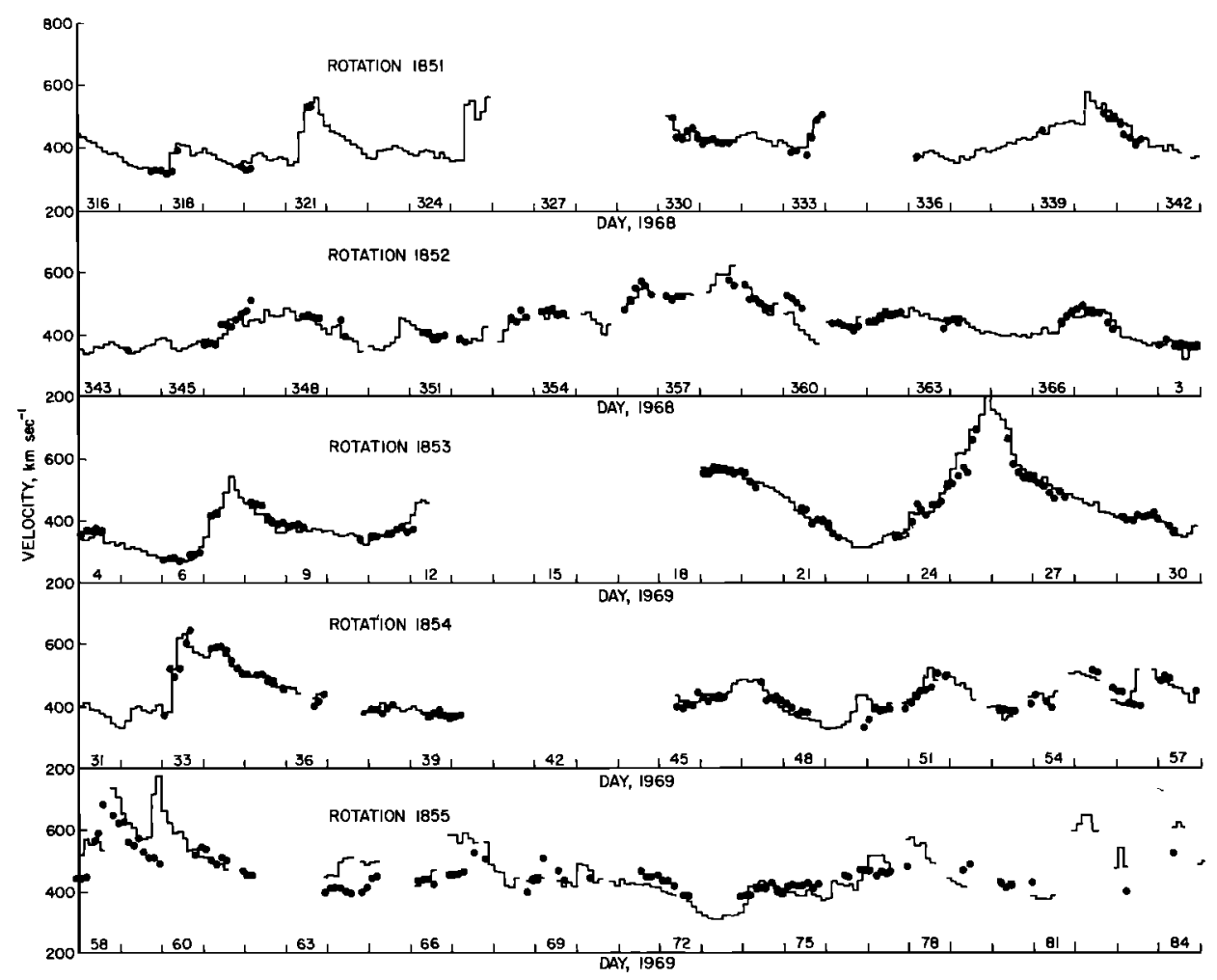

Fig. 2. The 3-hour averages of the solar wind velocity observed at Pioneer 9 (bars) and at Ogo 5 (circles) as a function of time at Pioneer 9. The Ogo 5 data were translated to Pioneer 9 time by using the 3-hour average of the velocity at Pioneer 9 and the corotation model (see text). 


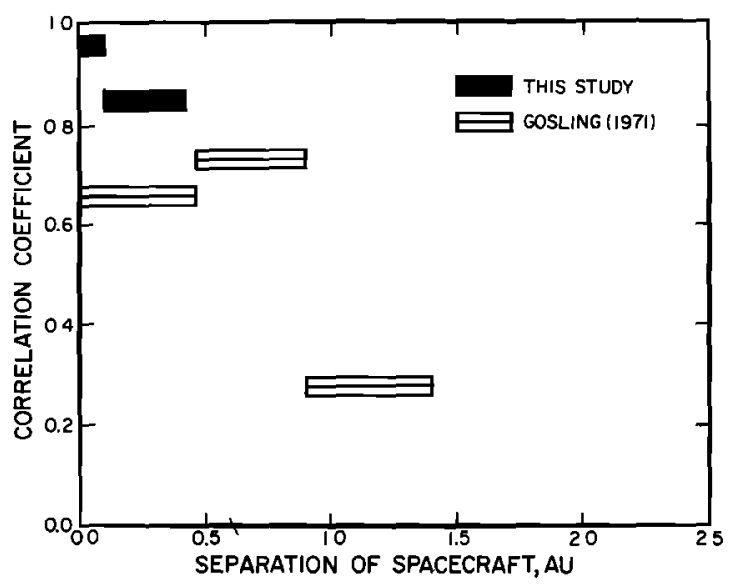

Fig. 3. The correlation coefficient of corotation pairs of velocity measurements as a function of the distance between two spacecraft. The correlation coefficients from this study (solid bars) and from Gosling [1971] (open bars) are shown. The separation between the spacecraft in Gosling's study was mainly azimuthal (see text).

spherically symmetric model in which the last term of (1) is neglected, this particular high-velocity stream would have reached 1 AU during a data gap at Ogo.

The general trend shown by Figure 2 and by part 4 of Table 1 is for the difference between the velocities at Ogo and Pioneer to increase with increasing distance between the two spacecraft due to temporal and spatial inhomogeneities of the solar wind. Figure 3 is a graph of the dependence of the correlation coefficient of corotation pairs of velocity measurements on the distance between the two spacecraft. The two highest correlation values come from this study of Ogo 5 versus Pioneer 9 observations where the separation between the two spacecraft is at times both radial and azimuthal (see Figure 1). The three lowest values were obtained by Gosling [1971] by using the daily quick-look velocities [SolarGeophysical Data, 1970; Intriligator, 1974] from Pioneer 6 and Pioneer 7 where the separation between Pioneer 6 and 7 is mainly azimuthal.

Acknowledgments. This work was sponsored by NASA grant NGR 05-002-165, NGR 05-002-59, NGR 05-018-181, contract NAS7-100, and by grant PF-021 President's Fund Caltech.

The Editor thanks A. Egidi and J. T. Gosling for their assistance in evaluating this report.

\section{References}

Collard, H. R., and J. H. Wolfe, Radial gradient of solar wind velocity from 1 to $5 \mathrm{AU}$, in Solar Wind Three, edited by C. T. Russell, University of California at Los Angeles, Los Angeles, 1974.

Gosling, J. T., Variations in the solar wind speed along the earth's orbit, Solar Phys., 17, 499, 1971.

Graham, R. A., and F. E. Vescelus, Ogo-E plasma spectrometer, Proc. Nat. Instrum. Soc. Amer. Aerosp. Instrum. Symp. 13th, 111, 1967. Intriligator, D. S., The reliability of the daily quick-look solar wind velocities as indicators of interplanetary activity, J. Geophys. Res., 79, 2491, 1974.

Intriligator, D. S., J. H. Wolfe, D. D. McKibbin, and H. R. Collard, Preliminary comparison of solar wind plasma observations in the geomagnetospheric wake at 1000 and 500 earth radii, Planet. Space Sci., 17, 321, 1969.

Neugebauer, M., Initial deceleration of solar wind positive ions in the earth's bow shock, J. Geophys. Res., 75, 717, 1970.

Neugebauer, M., Computation of solar wind parameters from the Ogo 5 plasma spectrometer data using hermite polynomials, Tech. Memo. 33-519, Jet Propul. Lab., Pasadena, Calif., 1971.

Neugebauer, M., and C. W. Synder, Mariner 2 observations of the solar wind, 1, Average properties, J. Geophys. Res., 71, 4469, 1966.

(Received February 13, 1973; accepted December 3, 1974.) 